Revue d'histoire de l'Amérique française

REVUE D.HISTOIRE DE L'AMÉRIQUE FRANÇAISE

\title{
La pédagogie du sexe : un aspect du discours catholique sur la sexualité au Québec (1930-1960)
}

\section{Gaston Desjardins}

Volume 43, numéro 3, hiver 1990

URI : https://id.erudit.org/iderudit/304814ar

DOI : https://doi.org/10.7202/304814ar

Aller au sommaire du numéro

Éditeur(s)

Institut d'histoire de l'Amérique française

ISSN

0035-2357 (imprimé)

1492-1383 (numérique)

Découvrir la revue

Citer cet article

Desjardins, G. (1990). La pédagogie du sexe : un aspect du discours catholique sur la sexualité au Québec (1930-1960). Revue d'histoire de l'Amérique française, 43(3), 381-401. https://doi.org/10.7202/304814ar
Résumé de l'article

Dans le Québec des années 1930-1960, le discours catholique sur la pédagogie sexuelle s'articule autour de trois composantes majeures : l'éducation de la chasteté, l'ennoblissement de la sexualité et la pédagogie "nouvelle ». Diverses tendances s'affrontent et s'enchevêtrent; entre les pièces usées du vieux dispositif s'insinuent et se renforcent des idées neuves. Les différentes problématiques s'inscrivent dans un renouvellement des perceptions de la famille. Dans l'après-guerre, malgré la présence de structures d'encadrement caduques, les intervenants se font plus nombreux et variés. En suivant les lignes de transformation du discours, on perçoit l'ébauche d'un dispositif pédagogique moderne.
Tous droits réservés @ Institut d'histoire de l'Amérique française, 1990
Ce document est protégé par la loi sur le droit d'auteur. L'utilisation des services d'Érudit (y compris la reproduction) est assujettie à sa politique d'utilisation que vous pouvez consulter en ligne.

https://apropos.erudit.org/fr/usagers/politique-dutilisation/ 


\title{
LA PÉDAGOGIE DU SEXE: UN ASPECT DU DISCOURS CATHOLIQUE SUR LA SEXUALITÉ AU QUÉBEC (1930-1960) ${ }^{1}$
}

\author{
GASTON DESJARDINS \\ Département d' histoire \\ Université du Québec à Montréal
}

\begin{abstract}
RÉSUMÉ
Dans le Québec des années 1930-1960, le discours catholique sur la pédagogie sexuelle s'articule autour de trois composantes majeures: l'éducation de la chasteté, l'ennoblissement de la sexualité et la pédagogie «nouvelle». Diverses tendances s'affrontent et s'enchevêtrent; entre les pièces usées du vieux dispositif s'insinuent et se renforcent des idées neuves. Les différentes problématiques s'inscrivent dans un renouvellement des perceptions de la famille. Dans l'aprèsguerre, malgré la présence de structures d'encadrement caduques, les intervenants se font plus nombreux et variés. En suivant les lignes de transformation du discours, on perçoit l'ébauche d'un dispositif pédagogique moderne.
\end{abstract}

\section{ABSTRACT}

In Quebec between 1930 and 1960, Catholic discourse regarding sexual education revolved around three major themes: the teaching of chastity, the ennobling of sexuality and the "new" pedagogy. Various tendencies confronted each other and inter-mingled; within the confines of the old system, new ideas emerged and grew in strength. These differing approaches can be linked to the changing perceptions of the family. In the post-war years, despite an outdated institutional framework, participants in this debate became more numerous and more varied. Tracing the ways in which this discourse was transformed sheds light on the emergence of a modern pedagogical system.

\section{INTRODUCTION}

Parler du discours catholique sur la pédagogie sexuelle au Québec, pour les années 1930 à 1960 , c'est, selon une opinion répandue, se condamner à répéter ce que tout le monde sait: le péché de la chair fut «le grand péché du Québec», le symbole de notre enfermement national. Dans la foulée libératrice des années 1960, quiconque se voulait moderne ne devait-il pas y aller de ses accusations ou de ses sarcasmes

1 Cet article est tiré de La pédagogie du sexe: un aspect du discours catholique sur la sexualité au Québec (1930-1960), mémoire de maîtrise (histoire), Université du Québec à Montréal, $1985,156 \mathrm{p}$. 
contre son passé oppressif, contre l'éducation puritaine, le climat de peur et de frustration de sa sexualité enfantine? Mais, curieusement, un examen plus approfondi des attitudes par rapport à la sexualité révèle une certaine permanence, à travers les décennies, de ce thème d'une «intoxication» répressive, toujours imputée au puritanisme de la génération précédente. Ainsi, dans son ouvrage d'éducation sexuelle publié en 1930, le docteur L.-P. Mercier exposait les éléments d'une perception qui sera réitérée, avec plus ou moins de vigueur, tout au long de la période.

L'éducation sexuelle. Cette question aurait été considérée bien osée, il y a vingt ans, par la majorité des parents. Elle l'est beaucoup moins aujourd'hui mais, dans l'esprit d'un trop grand nombre, les préjugés contre la franchise en ce sens existent encore malheureusement; résultat d'une fausse pudeur développée chez nous par le silence de leurs parents. À ceux-là, il va falloir commencer par prouver la nécessité d'une telle éducation. ${ }^{2}$

Dès le début des années 30 , dans les milieux catholiques québécois, on reconnaissait donc, d'une manière plus ou moins résolue, l'importance d'une éducation sexuelle pour les jeunes. Au stade de l'enfance ${ }^{3}$, au moment où la puissance diffuse de l'instinct sexuel apparaissait à la fois riche et dangereuse, comment allait-on considérer ce potentiel en devenir? Fallait-il insister sur l'assimilation d'un code d'interdits ou mettre l'accent sur une éducation plus formatrice? Ne seraiton pas mieux avisé de considérer des méthodes adaptées aux exigences sociales modernes? À toutes ces questions, les réponses furent diverses. Mais qui prétendait donc être en droit d'exercer le contrôle sur la sexualité de l'enfant? Comment et à partir de quels critères? Quels enjeux sous-tendaient les différents types d'intervention? À travers les considérations sur l'enfance passent les anticipations diverses d'une société future. Et la pédagogie de la sexualité s'avère un domaine stratégique à travers lequel chacun propose ses valeurs et ses espérances sociales. Derrière les besoins de l'enfant se profile aussi la promotion de critères de réussite familiale.

Discours catholique ne doit pas s'entendre ici comme l'apanage exclusif du catholicisme officiel, celui du magistère et de ses interprètes autorisés. Le discours catholique sur la sexualité est multiple. Il s'élabore à travers un ensemble d'écrits se proposant de défendre et de renouveler le système prescriptif en matière de pédagogie sexuelle. Les tendances y sont variées et contradictoires.

$2 \quad$ L.-P. Mercier, Quoi dire, comment dire et quoi faire. Éducation sexuelle (Montréal, Éd. Valiquette, 1930), 15.

La différence enfance-adolescence à travers les textes reste bien difficile à départager. La distinction ne devient précise que lorsqu'on aborde explicitement le sujet de ce đépartage. Pour le reste, on demeurera prudent. Rappelons que les enseignements de Pie XI et de Pie XII permettaient de traiter de sexualité entre parents et adolescents, mais restaient relativement réticents lorsqu'il s'agissait des plus jeunes. 
J'essayerai de voir comment s'articule le débat. Cela implique une révision de la représentation courante d'une morale catholique de la sexualité apparaissant au Québec comme un tout à peu près constant, homogène et singulièrement répressif. Je crois avisé de la considérer plutôt comme partie intégrante d'un ensemble fort complexe, en transformation, composant sans cesse avec le développement du savoir, avec la modification des rapports de pouvoir et avec des exigences de contrôle et d'encadrement de plus en plus élaborées.

Une telle étude fait appel à une documentation éparse, à l'exploration d'un large corpus regroupant des textes issus de milieux variés. Pour cet article, j'ai retenu un ensemble de monographies, brochures, articles et textes divers représentant un éventail des tendances catholiques québécoises de la période. Celles-ci se regroupent en deux catégories. D'une part, les auteurs dont le but est la diffusion et l'explication de la ligne directrice officielle en matière de sexualitét ${ }^{4}$. D'autre part, les intervenants qui ont cherché à évaluer la pertinence de ce système prescriptif rigoriste. Ces derniers, tout en se réclamant des grandes valeurs du catholicisme, s'appliquent à renouveler le système normatif et l'éthique de la sexualité 5 .

Dans le Québec des années 1930-1960, le discours catholique sur la pédagogie sexuelle s'articule autour de trois composantes majeures: l'éducation de la chasteté, l'ennoblissement de la sexualité et la pédagogie «nouvelle». J'entends examiner comment les diverses tendances se sont profilées, comment, à travers les affrontements multiples, les attitudes se sont conjuguées.

\section{1 - PÉDAGOGIE DE LA CHASTETÉ}

Le problème de la préservation morale des enfants, élément important de la pastorale chrétienne, suscite une attention particulière autour des années 1930. Devant la diffusion de plus en plus envahissante d'un certain «naturalisme pédagogique», dont la prétention à l'objectivité scientifique semble mettre à distance les préoccupations morales, le magistère de l'Église cherche à réitérer sa ligne directrice en matière de pédagogie sexuelle ${ }^{6}$. Dans les milieux catholiques québécois, ce point de vue officiel ${ }^{7}$ se traduit d'abord par une «pédagogie de la chasteté».

4 Un discours caractéristique des publications Nos Cours, L'École sociale populaire, Les Semaines sociales du Canada, Relations, etc. Cette attitude se manifeste aussi, de façon diffuse ou spécifique, dans nombre de textes traitant de pédagogie, de morale et de la famille. Un repérage exhaustif de ce genre d'écrits n'est pas indispensable; les traits dominants sont sans cesse réitérés à travers des prescriptions multiples. On peut parler, pour la période, d'un noyau dogmatique avec ses effets de redondance et de saturation caractéristiques.

5 Voir surtout Collège et famille, L'École des parents, L'Enseignement secondaire, L'Enseignement primaire, La Famille, Service social, Revue dominicaine.

6 Pie XI, «Lettre encyclique sur l'éducation chrétienne de la jeunesse», dans Paul Foulquié, L'Église et l'école (Paris, SPES, 1977), 139-187.

7 Voir, entre autres, Nos cours, Relations, École sociale populaire, Semaines sociales du Canada, les traités de morale et les catéchismes. 
Sur la base des grands principes de la tradition chrétienne en matière de sexualité (idéal ascétique, impératifs de reproduction, etc.), deux tendances pédagogiques se superposent, laissant ressortir une variation de ton: a) la stratégie du silence, b) l'éducation de la chasteté.

\section{A - Stratégie du silence}

Aux yeux de plusieurs, tout ce qui concerne la pédagogie sexuelle au Québec avant 1960 entre facilement sous un seul vocable: conspiration du silence. Effectivement, c'est l'impression qui ressort de nombre de directives générales ${ }^{8}$. Mais, dans la pratique, s'agit-il vraiment d'une consigne du silence? N'a-t-on pas le silence un peu loquace? Par exemple, dans cette énumération de péchés devant servir à faire un examen de conscience:

Regarder de mauvaises choses ou de mauvaises images; dire de mauvaises paroles; entretenir de mauvaises pensées; toucher de mauvaises choses sur soi ou sur d'autres; faire de mauvaises choses seul ou avec d'autres. ${ }^{9}$

Voilà des «choses» qu'on n'en finit plus d'évoquer, mais dont on ne veut pas vous parler. Quoi de plus intrigant! Sans doute suscite-t-on des questions, éveille-t-on des curiosités. Erotisme du confessionnal et de la pastorale? Peut-être. Mais bien davantage une incitation au travail sur soi par une éducation graduelle au signe de la concupiscence: ces sensations, lorsqu'elles viendront assaillir votre conscience, vous devez savoir les identifier comme péché et, au moindre soupçon de complaisance, venir affranchir votre conscience auprès de votre confesseur. C'est donc dans cet espace ambigu, à travers un double effet de curiosité et de suspicion, que viennent se placer la confession et la direction spirituelle $^{10}$.

\section{B - Éducation de la chasteté}

De plùs en plus, cependant, l'Église est forcée de considérer les implications d'une «éducation sexuelle». Elle ne peut, en effet, minimiser la menace de certains discours qui s'alimentent au savoir nouveau et qui prétendent mieux correspondre aux réalités modernes ${ }^{11}$. Ainsi, à

8 Voir, par exemple, Mgr F.-X. Ross, «L'enseignement religieux», L'École sociale populaire, 196 (1930): 9; Rosario Fontaine, «Le freudisme», L'Union médicale du Canada, 60,6 (juin 1931): 432-433. Ce type de recommandation était encore relativement répandu dans les années 1930.

9 J.-A. Charlebois, Catéchisme préparatoire à la première communion (Montréal, Les Clercs Saint-Viateur, 3e édition, 1934), 59.

${ }_{10}$ Entre autres, Maurice-H. Beaulieu, «Direction spirituelle», L'Enseignement secondaire, 24, 2, 3, 5 et 8, (1944 et 1945); Victorin Germain, Catéchisme des 6ème et 9ème commandements de Dieu (Québec, Action catholique, 2e édition, 1938).

11 Par exemple, M.-M. Bergeron, o.p., «Préservation morale des enfants», Revue dominicaine (1936): 215-223; A. Bissonnette, «D'où vient la crise morale?», Revue dominicaine, 35 (1929): 31-41. 
partir des années 1930 au moins, la méthode appelée «consigne du silence» ne fait plus recette et apparaît même comme plutôt néfaste, c'est-à-dire susceptible d'exposer à de "graves mécomptes» dans une société où «les dangers d'une éducation malsaine se multiplient pour ainsi dire à l'infini»" ${ }^{12}$. Dorénavant, une intervention en ce domaine s'avère indispensable. Les propagateurs du catholicisme officiel, faisant écho au magistère de Pie XI, rejettent toutefois les méthodes «pernicieuses» et «subversives» de ladite éducation sexuelle, pour s'employer plutôt à promouvoir une «éducation de la chasteté»" ${ }^{13}$.

Mais, fondamentalement, l'embarras reste le même. Peut-on donner «une vision claire de cette vertu», c'est-à-dire être explicite sur le sujet, sans risquer de déclencher les mécanismes de la complaisance? Il paraît impossible de sortir le sexe du confessionnal sans altérer la substance même du pouvoir de l'Église en matière de sexualité. Pour contourner la difficulté, et puisqu'il faut éduquer, on agira de manière indirecte.

Éduquer les jeunes dans le sens de la pureté, c'est avant tout former leur caractère ou leur volonté, puis les renseigner d'une façon délicate et adaptée à leurs réels besoins, enfin c'est imprégner cette formation du coeur et de l'esprit d'une atmosphère surnaturelle. ${ }^{14}$

Il faut amener l'enfant dès l'âge «le plus tendre» à acquérir des habitudes de rigueur et de vertu. Cela implique, d'une part, une prophylaxie morale encadrée par les techniques traditionnelles et, d'autre part, l'initiation graduelle à un ordre social bien spécifique. Autorité, hiérarchie et renoncement sont compris comme conditions essentielles à une véritable promotion sociale, avec, en filigrane, des types d'éducation différenciés pour filles et garçons. En théorie, il s'agit moins de chercher à écraser les manifestations de la sexualité sous une avalanche d'interdits que d'essayer d'établir davantage de rapports entre les principes moraux et la possibilité de «réussite sociale».

[...] la chasteté, gardienne de la foi, fortifie tout ce qui fait la valeur de la vie: «la vaillance, le courage, la joie dans le travail». [...] Pour assurer le succès, les parents développent chez [l'enfant] le goût de la lutte qui mène à la victoire. [...] Il ne reste plus qu'à utiliser deux précieux facteurs de succès matériel et scolaire: l'enthousiasme et la joie. ${ }^{15}$

\footnotetext{
12 Georges Levasseur, «Éducation de la pureté chez les jeunes», Nos Cours, 6,8 (novembre

d'écrine tendance qui s'exprime surtout dans les revues Nos Cours et Relations et nombre d'écrits cherchant explicitement à diffuser les enseignements du magistère.

14 Georges Levasseur, loc. cit., 1. Voir aussi A.-L. Barthélémy, o.p., «L'éducation», L'École sociale populaire, 36 (1930): 75; Maurice-H. Beaulieu, loc. cit.; Victorien Germain, Le point d'interrogation; exposé sommaire du mystère de la génération humaine et des questions connexes, rédigé à l'intention des mères perplexes et de leurs filles questionneuses (Québec, L'Action catholique, lère édition, 1936), 270 p.

15 Georges Levasseur, loc. cit., 1.
} 1944): 1 . 
Dans ce type de discours, on conçoit tout naturellement que les parents soient les premiers responsables de l'éducation de leurs enfants. En pratique, cependant, ils ne semblent pas vraiment «autorisés» à traiter de sexualité avec leurs enfants d'une manière autonome, du moins pas avant l'adolescence ${ }^{16}$. Pour les autres éducateurs laïcs, l'unique biais d'intervention accepté est l'introduction au code moral, à la réussite et à l'affirmation sociale en accord avec les valeurs chrétiennes ${ }^{17}$.

Ainsi, la direction spirituelle et le sacrement de pénitence restent les outils privilégiés, et pratiquement exclusifs, par lesquels non seulement on peut aborder explicitement la sexualité, mais aussi sonder l'âme de façon méthodique. L'enfant doit s'avouer en offense à luimême et au monde; il apprend à modeler sa conscience, à se construire une âme capable d'asservir le corps et d'y extirper le mal par la force de la volonté. Dans ces lieux priviligiés, intimes, on veut, dans un secret rapport au sexe, faire de l'enfant pénitent un bon «sujet moral» et, subséquemment, un bon «sujet social».

Le rejet par Pie XI des méthodes dites «d'éducation sexuelle» peut s'interpréter en regard de ces considérations. L'appellation elle-même, par sa référence trop crue au sexuel, s'attaque au «tabou» et risque ainsi de renverser un élément indispensable des techniques traditionnelles de l'Église. «L'Église garde jalousement ses lois morales, les applique, avec méthode et charité, et ne pactise pas avec les erreurs que la passion soulève. ${ }^{18} \mathrm{Au}$ contraire, par le terme «éducation de la chasteté», on réitère une position globalisante qui rend toute considération sur le vécu sexuel inséparable de l'enseignement catholique traditionnel. La nuance est capitale: tout un mode de régulation de la sexualité est en cause et, par le fait même, toute une hiérarchie, toute une structure de pouvoir ${ }^{19}$.

Cette approche traditionnelle se manifeste de façon bruyante et persistante durant les trois décennies étudiées ici, mais on peut y voir aussi une attitude défensive ${ }^{20}$. À partir des années 1930, les tenants de ce discours redoublent d'efforts pour valoriser les principes de chasteté à la fois comme barrière à la dégénérescence et comme seule garantie de l'ordre social et des valeurs fondamentales. On s'acharne, d'une part, à scander les impératifs d'une chaste éducation en référence à un univers symbolique et à un savoir sur le corps de plus en plus désuet et, de l'autre, on fait flèche de tout bois pour contrer les tendances nou-

16 Pie XI, loc. cit.

17 Maurice-H. Beaulieu, loc. cit.

18 A. Bissonnette, o.p., loc. cit., 41 .

19 De nombreux ouvrages ont traité de cette structure de pouvoir. Voir Jean Hamelin et Nicole Gagnon, Histoire du catholicisme québécois. Le XXème siècle (Montréal, Boréal Express, 1984), 2 vol.

${ }_{20}$ Voir entre autres A. Bissonnette, loc. cit.; Maurice-H. Beaulieu, loc. cit.; Lettre pastorale des évêques, «Croisade de la pureté» (1946), dans Mandements et lettres pastorales (Montréal, Arbour et Dupuis, 1952), 20: 210-240. 
velles à la valorisation des corps et du plaisir qui viennent jeter le doute sur la pertinence de l'enseignement de l'Église ${ }^{21}$.

\section{2 - ENNOBLISSEMENT DE LA SEXUALITÉ}

Ainsi, dans le sillage des vérités doctrinales se maintient tout un système prescriptif. Mais, peu à peu, un nombre grandissant de nuances, voire de divergences viennent ébranler le noyau dogmatique. Car, dans les milieux catholiques, plusieurs intervenants notoires, principalement de France et de Belgique, manifestent une volonté d'ajuster la pastorale aux phénomènes sociaux modernes. Mgr Jean Vivier, futur archevêque de Paris, écrit en 1929:

[...] il serait douloureux et humiliant pour notre pauvre humanité de soutenir que le domaine où jaillissent les sources de la vie est le seul qui doit rester fermé à tout progrès pédagogique et même scientifique $[\ldots]$. Nous croyons que les initiations claires faites avec le tact voulu doivent être regardées comme une obligation grave $[\ldots]^{22}$

Et les catholiques du Québec ne sont pas en reste:

Par la psychologie et la physiologie, nous savons aujourd'hui les vérités naturelles du sexe si intimement liées à la santé physique, mentale et morale que les ignorer sciemment serait pêcher par négligence dangereuse, pour ne pas dire criminelle. ${ }^{23}$

L'ignorance des questions qui se rattachent à la différence des sexes, aux mystères de la vie et aux impulsions de la sensibilité naissante, constitue-t-elle, dans notre milieu social, un péril si évident qu'il faille y parer par une initiative hâtive et une instruction scientifique? Il est clair que le problème se pose, en notre pays comme partout ailleurs, avec une fulgurante évidence. Cette initiation précoce, qu'on la nomme ainsi à tort ou à raison, il faut la faire. ${ }^{24}$

Les articles et les manuels traitant de l'enseignement de la sexualité aux enfants se multiplient à partir des années 1940. On y retrouve, avec des impulsions plus ou moins «modernistes», des membres du clergé, des éducateurs laïcs, des parents, des médecins, des interve-

21 On peut relier ce discours aux angoisses du pouvoir clérical devant les transformations sociales accélérées, mais il faut y inclure l'impact du savoir sur le corps et la sexualité. Voir, par exemple, Hector Palardy, L'hygiène sexuelle (Chicoutimi, Impr. du Saguenay, 1922); Hervé Trudel, Élements de morale médicale (Montréal, Granger, 4e édition, 1940). Certaines publications sur la sexualité, provenant des milieux anglophones protestants, et diffusées assez largement dans les premières décennies du XXe siècle, ont pu influencer les écrits catholiques québécois; voir Michael Bliss, «Pure Books on Avoided Subjects», Communications historiques (1970): 89108. 1930), 7-8.

23 L.-P. Mercier, op. cit., 23.

24 M.-M. Bergeron, o.p., loc. cit., 215. 
nants des milieux judiciaires, des vulgarisateurs, etc. Mentionnons les ouvrages du docteur L.-P. Mercier, de Victorin Germain, de Jean Bousquet, d'Odette Vincent-Fumet et de Roméo Meloche ${ }^{25}$. Soucieux d'adapter les références normatives au contexte social en transformation, ces spécialistes ont le sentiment profond de répondre à un besoin depuis longtemps ressenti. Certains ouvrages bénéficient d'un succès populaire assez important ${ }^{26}$. Cependant, le ton nuancé, les lettres d'appui et les cautions diverses placées en avant-propos témoignent de l'importance de se présenter comme qualifié et, surtout, de se mettre à l'abri des foudres de l'orthodoxie ${ }^{27}$.

Contrairement aux discurs précédents, on aborde, dans ces écrits, autant les aspects anatomiques et psychologiques que le côté moral du problème. On veut se démarquer des attitudes pédagogiques trop puritaines des générations précédentes, ennoblir la procréation aux yeux des jeunes et ramener plus explicitement aux parents la tâche d'instruire leurs enfants. De la «consigne du silence», on passe au «silence coupable». «Ah! Quelle folie que ce silence, dira Jean Bousquet, quelle exécrable et inqualifiable folie! Et quelle lâcheté! ${ }^{28}$

Ces manuels, qui s'adressent d'abord aux parents, recommandent une pédagogie adaptée à chaque étape de l'enfance et de l'adolescence. L'éducation sexuelle doit débuter dès que l'enfant commence à poser des questions. Quelques auteurs, comme Meloche et Germain, abordent les fonctions sexuelles de façon passablement détaillée. Les explications qui se veulent «simples» et «franches» font appel à une connaissance assez élaborée de l'anatomie. Dissipant quelques préjugés sur les menstruations ou sur les pollutions nocturnes, on taxe, par contre, d'infamie les comportements dits anormaux comme la masturbation ou l'homosexualité.

Dans l'ensemble, ces informations sont systématiquement orientées vers la procréation dans le mariage. Les divers stéréotypes masculins et féminins y sont exposés: caractéristiques psychologiques, rôles sociaux et complémentarité «naturelle» des deux sexes. Le mode de régulation proposé s'appuie sur un appel à la raison. Amour raison-

25 L.-P. Mercier, Quoi dire, comment dire et quoi faire ... (1930); Victorin Germain, Le point d'interrogation ... (1936); Jean Bousquet, o.p., Comment éduquer vos enfants (Montréal, Presses dominicaines, 1942); Odette Vincent-Fumet, Comment le dire? Nos petits et les mystères de la vie (Montréal, s.é. 1945); Roméo Meloche, Parlons à nos enfants (Montréal, Éd. de la Gerbe de blé, 1948) et La vie ... quelle merveille! (Montréal, Éd. de la Gerbe de blé, 2e édition, 1952).

26 Parlons à nos enfants de Roméo Meloche aurait atteint 70000 exemplaires, si on en croit l'auteur dans l'avant-propos de La vie ... quelle merveille!.

${ }_{27}$ L'abbé V. Germain, par exemple, se fera hésitant à bien des occasions. Sa difficulté, c'est de trouver le ton juste, de concilier les grands principes moraux énoncés par l'orthodoxie catholique (dont il est le propagateur) avec sa volonté de se débarrasser des considérations trop culpabilisantes. Voir Le point d' interrogation ...

28 Jean Bousquet, o.p., op. cit., 86. 
nable, maîtrise des passions, noblesse d'âme permettent, dit-on, d'atteindre un statut supérieur.

Les pauvres victimes trop faibles pour résister aux attraits du plaisir de la chair en viennent à n'avoir que mépris pour l'amour, la justice, la pureté et le désintéressement. Elles ne peuvent plus connaître les joies supérieures de l'esprit, ni éprouver le contentement occasionné par les belles actions et les nobles pensées. ${ }^{29}$

En somme, ce travail individuel sur la volonté est orienté vers un certain ordre social répondant des valeurs fondamentales (famille, travail, ordre, vertu, etc.); l'aide d'un conseiller, issu du milieu familial, est fortement suggérée ${ }^{30}$.

Plusieurs de ces ouvrages, publiés dans les années 1940 et 1950 , abordent explicitement ce principe selon lequel une intervention plus adéquate sur la sexualité des enfants implique un déplacement - au moins partiel - des mécanismes de contrôle vers l'intérieur de la cellule familiale. C'est le cas par exemple du médecin Lucien Royer qui, en 1945, conçoit le mode d'intervention axé sur l'intensification des rapports familiaux comme un renversement $d$ 'attitude ${ }^{31}$. D'après lui, les appels à la crainte et à la prudence d'un certain discours «traditionnel» n'ont pas de véritable prise sur «les jeunes imaginations». L'expérience a «démontré combien ces considérations sont impuissantes à freiner les investigations d'une sensualité surexcitée». Plutôt que «du dehors», il faut s'appliquer à agir «du dedans», «à la source des volitions», c'est-à-dire sur les désirs et l'imagination même de l'enfant ${ }^{32}$. Royer renverse aussi la méthode ancienne: «ce ne sont pas les laideurs de l'impureté et de ses suites qu'il faut présenter à l'esprit vierge, c'est la beauté des lois de la vie dans l'univers et dans l'humanité. ${ }^{33}$ Cet enseignement préventif, selon l'auteur, doit à tout prix être donné avant la puberté, afin de munir l'enfant d'un système de défense indispensable pour affronter cette «époque troublée».

La distinction principale de ce discours, par rapport à celui étudié précédemment, tient surtout dans l'affirmation d'une «direction» familiale plutôt que pastorale en matière de pédagogie sexuelle. C'est la famille qui devient la cellule d'intimité et de confidence. Désormais, comme le souligne Odette Vincent-Fumet, il appartiendra aux parents «de préparer eux-mêmes le terrain [...], de surveiller de jour en jour l'évolution de la pensée en germe» ${ }^{34}$. La méthode pédagogique doit

29 Roméo Meloche, La vie ... quelle merveille!, 169.

30 Voir les ouvrages de Mercier, de Meloche et de Vincent-Fumet.

31 Lucien Royer, Autour de l'être féminin (Montréal, Éd. de l'étoile, 1945), 150 p., et Autour de l'être masculin (Montréal, Éd. de l'étoile, 1945), 150 p.

32 Lucien Royer, Autour de l'être féminin, 55-63.

33 Ibid., 56.

34 Odette Vincent-Fumet, op. cit., 16. 
circonscrire et désamorcer les zones d' «intimité négative», en s'appuyant sur le principe que le secret fait souffrir, qu'il est dangereux de s'enfermer à l'intérieur de soi.

Promets de toujours poser des questions [...], ouvre bien ton âme [...]. Renseigne-toi. Ne va pas constituer autour de tes problèmes et inquiétudes une zone brumeuse secrète où personne ne pourra pénétrer et qui te fera souffrir. Ne t'enferme pas en toi-même. ${ }^{35}$

Ainsi faut-il amener l'enfant à se confier pour se délivrer de ses angoisses et à s'affirmer en accord avec la raison sociale.

L'éducation sexuelle a donc pour mission au début non de «mettre en garde» l'enfant, mais de l'instruire à la lumière pure qui sera son préservatif, il la fera briller en lui par une initiation graduelle à un idéal de vie qui ennoblisse à ses yeux tout le domaine du sexe et de ses lois. ${ }^{36}$

Une saine éducation sexuelle suppose un langage approprié, un vocabulaire juste (scientifique) et sérieux; les échanges entre compagnons et compagnes sont rigoureusement prohibés. Pour les filles, la démarche devra être «plus poétisée», "plus sentimentale que physique». En général, pour les jeunes enfants, les deux parents doivent s'impliquer; mais, à l'approche de l'adolescence, c'est la mère qui s'occupera de sa fille tandis que le père suivra le garçon. Dans l'ensemble de ces textes, on constate une difficulté persistante: la hantise du mot juste, la peur de troubler l'esprit fragile de l'enfant. Comme l'écrit Odette Vincent-Fumet: «Chaque fois j'ai répandu la vérité avec le souci, presque l'angoisse, de choisir les mots appropriés pour ne pas déformer, pour ne pas abîmer, dans l'esprit de ma fille, l'idée qu'elle doit se faire de ce rôle magnifique qu'est la maternité. ${ }^{37}$ Un problème qui, une fois de plus, est attribué à l'éducation des générations précédentes: «nous avons été intoxiqués d'un esprit de fausse pudeur qui s'offusque des mots vrais au lieu d'en pénétrer l'idée» ${ }^{38}$, ajoute l'auteur.

En somme, on voit s'affirmer une tendance qui cherche à rompre avec un certain rigorisme traditionnel, et à prendre en compte de façon mieux adaptée au contexte moderne la responsabilité d'une éducation sexuelle. Sans laisser pénétrer les théories «dangereuses» pour l'ordre social chrétien, on considère indispensable de se ressourcer aux savoirs nouveaux (anatomie, biologie, psychologie, sociologie et même «sexologie») et d'éliminer les méthodes de contrôle culpabilisantes, néfastes à l'épanouissement de l'enfant.

\footnotetext{
35 Roméo Meloche, La vie ... quelle merveille!, 189-190.

Lucien Royer, Autour de l'être féminin, 57-58.

Odette Vincent-Fumet, op. cit., 6.

Ibid., 19.
} 
Tous ces défenseurs d'une attitude plus ouverte ne s'affranchissent pas entièrement, même les plus audacieux, des notions traditionnelles de péché et de chasteté. Le souci de dispenser une information vraie se fait contraignant, mais cette «nouvelle vérité» du sexe prête à équivoque et ces auteurs catholiques le savent bien. Obligés de composer avec les plus traditionnalistes, ils sont tiraillés et débordés par un nombre grandissant de théories divergentes ${ }^{39}$.

Le processus réclame une plus grande «conscientisation» et une éducation des parents: «il faut savoir, [il] faut un travail d'étude...» ${ }^{40}$, prévient-on. Ce que l'on veut toucher, ce sont d'abord les rapports parents-enfants; plus largement, c'est la famille, en tant qu'instrument renouvelé de régulation, qui est en cause.

Quand Roméo Meloche présente un manuel au style direct ${ }^{41}$, comme s'il s'adressait à l'enfant ou à l'adolescent, c'est bien un modèle de comportement qu'il veut proposer. De quel modèle est-il question? Lorsqu'on insiste tant sur la noblesse d'âme comme principe premier de régulation, lorsqu'on parle de maîtrise de soi, d'autonomie, d'idéal de réussite sociale, c'est bien à la famille en tant que «cellule autonome» que l'on s'adresse. Ce modèle familial mise sur des rapports plus intenses entre parents et enfants. L'enfant, dès le plus jeune âge, en relation intime avec son guide parental, apprendra à travailler sur lui. Il établira une correspondance étroite entre les pulsions qui se manifestent en lui et les valeurs inscrites dans le milieu environnant. La réussite de l'enfant est ainsi mise en correspondance avec la réussite familiale.

Cependant, ce n'est pas aux parents à définir les normes, il leur faut encore une fois référer à des autorités en la matière. Si le prêtre, dans cette catégorie de discours, cède du terrain, ce n'est pas vraiment pour laisser la place aux seuls parents. Ces derniers seront encadrés par un nombre grandissant de spécialistes: le titre de docteur (médecin, psychologue, pédiatre, psychiatre, etc.) apparaît de plus en plus distinctement comme une garantie de compétence.

\section{3 - TRANSFORMATION SOCIALE ET RENOUVEAU PÉDAGOGIQUE}

\section{A - Pour une pédagogie nouvelle}

Ce renouvellement des attitudes dans le domaine de la pédagogie du sexe doit être compris dans le cadre d'un mouvement plus large de

\footnotetext{
39 Voir les commentaires de Roméo Meloche, Parlons à nos enfants, 57-58.

77

40 Le Glaneur, «La famille devant l'enfant moderne», Collège et famille, 1,3 (mai 1944):

41 Roméo Meloche, La vie ... quelle merveille!
} 
renouveau pédagogique et en regard d'une transformation de la société québécoise ${ }^{42}$. La forte emprise des autorités ecclésiastiques sur le système d'éducation n'a pas empêché le travail en sourdine de certaines forces de changement. Au cours des années 40 , les «réformistes» marquent des points importants: loi de 1943 sur la fréquentation scolaire obligatoire; loi de 1946 «pour assurer le progrès de l'éducation»; réforme, en 1948, des programmes d'étude des écoles primaires élémentaires, etc. ${ }^{43}$

Peu à peu, les idées pédagogiques nouvelles ouvrent une brèche dans le rigorisme idéologique; plutôt que sur la formation du caractère, on met dorénavant l'accent sur le développement de l'intelligence. Tout en ménageant l'orthodoxie catholique, des leaders réformistes, tel Roland Vinette, cherchent à promouvoir une pédagogie qui mise sur l'intérêt, l'initiative, la liberté et les aptitudes des enfants ${ }^{44}$. D'autres écrits respectent également la personnalité de l'enfant et, à l'écoute des théories psychologiques et psychanalytiques, visent à exploiter les richesses plutôt que de s'acharner uniquement à écraser les dangers.

À la différence de l'attitude précédente, cette tendance novatrice se démarque de façon beaucoup plus nette des considérations morales défendues par le magistère de l'Église. Ses aspects fondamentaux sont débattus particulièrement dans des revues d'éducation comme Collège et famille, L'Enseignement primaire, L'École des parents, etc. ${ }^{45} \mathrm{Ce}$ projet de «renouveau pédagogique», même s'il ne fait pas directement allusion à l'éducation sexuelle, en contient toutefois des éléments en gestation.

Tout d'abord, on condamne les attitudes autoritaires, entraves à l'épanouissement des «potentiels» en devenir:

À l'âge où [pour] ses parents [...] la personnalité de l'enfant était méconnue, refoulée, inhibée, alourdie, tuée dès ses premières manifestations, le nôtre, notre enfant moderne, va, vient, furette, expérimente, $\mathrm{s}$ 'épanouit, affine ses sens au contact de la matière et cela dans le sens même des besoins de son développement. ${ }^{46}$

L'insistance soutenue sur la valorisation de soi est un autre aspect important. Comme élément premier de la force virtuelle de l'enfant, on évoquera l'individualité:

42 Voir L.-P. Audet, Histoire de l'enseignement au Québec (Montréal, Hurtubise HMH, 1971), 2 vol.; J.-L. Roy, La marche des Québécois (Montréal, Leméac, 1976), 383 p.

43 Louise Charpentier, Le programme et les manuels d'histoire de la réforme scolaire de 1948, mémoire de maîtrise (histoire), Université de Sherbrooke, 1983).

44 Roland Vinette, «L'école active; définition», L'Enseignement primaire (octobre 1944): 227-229 et «L'école nouvelle», L'Enseignement primaire (septembre 1945): 5-15.

45 Voir Claudine S. Vallerand, «Pour une pédagogie 'à la page'», Collège et famille, 2,3 (mai 1944): 81-86; Roland Vinette, «L'école active; définition», L'Enseignement primaire (septembre 1945): 5-15; Louis Bouchard, «La trempe du moi moral», Les Carnets viatoriens (1941): 23-26.

46 Claudine S. Vallerand, loc. cit., 81. 
[...] être soi-même en tout et partout, [voilà une] condition indispensable à toute vie féconde. Impossible de donner sa pleine mesure en rien, à moins de déployer sans contrainte les caractères qui sont le fond de la personne [...]. Dans l'ordre moral, l'homme qui n'est pas pleinement lui-même ne peut exercer une influence profonde dans aucun domaine. ${ }^{47}$

Plusieurs réformistes des années 1940 et 1950 craignent, dans cette perspective, que la discordance grandissante entre le code moral de l'orthodoxie catholique et les comportements effectifs ne rende l'assimilation des prescriptions de plus en plus superficielle ${ }^{48}$. Pour éviter le conformisme de façade, l'hypocrisie, il devient impératif de combler la distance entre la moralité de comportement et la perception de soi comme sujet moral ${ }^{49}$.

Telles sont, rapidement esquissées, les orientations réformistes des années 1940 et 1950 en matière de pédagogie. À travers ces élans, on reconnaît une appréciation beaucoup plus nette du potentiel de l'enfant. Les «méthodes scientifiques dans l'éducation» 50 deviennent indispensables au développement «normal» de la personnalité. Plusieurs publications diffusent ce savoir nouveau, répondant ainsi au besoin maintes fois exprimé de former des compétences pédagogiques mieux adaptées à la réalité québécoise ${ }^{51}$.

\section{B - Une affirmation plus diffuse de la pédagogie sexuelle}

Ce renouveau pédagogique ne donne pas immédiatement lieu à un véritable développement de la pédagogie sexuelle. Peut-être craint-on d'attirer les foudres de mouvements déjà vigoureusement impliqués dans les campagnes de «moralité publique» 52 . En fait, ce discours pédagogique novateur n'a sans doute pas d'assises suffisamment solides pour s'opposer directement, en matière sexuelle surtout, à un milieu éducatif encore fortement marqué par les méthodes traditionnelles. Pourtant, même si la plupart des manuels d'éducation sexuelle sont encore imprégnés d'orthodoxie, on remarque que les références aux savoirs modernes sur l'enfance et la pédagogie s'immiscent peu à peu entre les motifs strictement «moraux». La diffusion au Québec de publi-

47 Louis Bouchard, loc. cit., 24. Voir aussi Roland Vinette, «L'école nouvelle», L'Enseignement primaire (septembre 1945): 78 . 1: $359-363$

49 Louis Bouchard, loc. cit., 24-25; voir aussi Roland Vinette, «L'école nouvelle», L'Enseignement primaire (septembre 1945); Claudine S. Vallerand, loc. cit.

50 Hermas Bastien, Les méthodes scientifiques dans l'éducation (Ottawa, Les services éducatifs de la Légion canadienne, 1944), 18 p.

51 Voir J.-L. Roy, op. cit., 245-309.

52 Les campagnes de moralité publique dans l'immédiat après-guerre sont passablement bruyantes et dynamiques. Voir Gabriel Clément, Histoire de l'Action catholique au Canada (Montréal, Fides, 1972), 91-94. 
cations catholiques d'origine étrangère joue certainement ici un rôle ${ }^{53}$. Quelques thèmes issus de courants modernes émergent aussi dans les écrits de membres de la Société canadienne de psychanalyse, chez des conférenciers invités par l'École des parents, parmi les travailleurs sociaux ${ }^{54}$. On observe ces influences dans Collège et famille, Pédagogie-Orientation, L'École des parents, etc. Bref, des éléments de réflexion fleurissent dans le Québec des années 1950.

Ainsi, dans le développement des savoirs sur l'enfance et, surtout, avec la valorisation des théories psychanalytiques ${ }^{55}$, la sexualité apparaît de plus en plus comme le lieu premier où se joue le dépérissement ou l'épanouissement de la personnalité. De la juste appréciation d'un potentiel brut et de son encadrement dépendent le dynamisme ou l'étiolement des générations montantes ${ }^{56}$. Les carences de la famille dans l'éducation sexuelle sont susceptibles d'entraîner des névroses.
Satisfaire la curiosité de l'enfant par des explications détaillées peut provoquer chez lui une excitation précoce qui sera la source d'une anxiété intense. D'autre part, les parents, par leur silence, leurs hésitations embarrassées ou des gronderies, donnent souvent à l'enfant l'impression qu'il vient d'aborder un domaine mysté- rieux et tabou. Un sentiment de culpabilité naît chez lui, intensifié par l'attrait du fruit défendu. Il peut refouler toute curiosité tout en demeurant aux prises avec l'anxiété; ou il peut s'adonner à des pratiques sexuelles secrètes et souffrir d'un sentiment de culpabi- lité et du remords qu'elles entraînent. Dans les deux cas, nous avons une personnalité dont le développement est entravé. ${ }^{5 \zeta}$

La nécessité d'une éducation sexuelle plus adéquate se fait plus pressante. Lentement mais résolument, on délaisse la symbolique du péché originel comme principe premier de la prédisposition au «vice» pour adopter un langage plus «scientifique». L'objet majeur du débat n'est plus tant de savoir s'il faut accepter une pédagogie sexuelle que de fixer les modalités de cette pédagogie. Et les interrogations premiè-

53 Les références (critiques, allusions, mentions bibliographiques) à des publications étrangères sont fréquentes. Par exemple, André Berge, L'Éducation sexuelle chez l'enfant (Paris, Presses universitaires de France, coll. "Que sais-je», 1952), 125 p., et de nombreux articles publiés dans L'École des parents (France); André Arthur, Les mystères de la vie expliqués aux enfants (Paris, Éditions ouvrières, 1958), 48 p.; les carnets Docteur que faire?, édités par le Centre médico-familial de Bruxelles; les écrits de Marc Oraison du Centre d'études Laennec; sans parler des ouvrages de toutes origines qui ne manqueront pas de susciter des remous, comme les rapports Kinsey.

54 Par exemple, J.-B. Bélanger, «La psychanalyse», L'Union médicale du Canada (mars 1956): 304-311; Paul L'Archevêque, "Les enfants trop connaissants pour leur âge», PédagogieOrientation (février-avril 1950): 9-21.

55 Soeur Agnès-de-Marie, «Anna Freud et la psychothérapie des enfants», PédagogieOrientation, 7 (1953): 50-79.

56 Opinion passablement répandue, dont les observations les plus «modernes» se retrouvent dans L'Union médicale du Canada et dans la revue Service social. 235-236. 
res persistent: à qui imcombe cette responsabilité? Comment devraiton procéder? À partir de quelles références normatives?

À la fin des années 1950, le caractère général du discours catholique se traduit par une préparation de l'enfant à ses fonctions «d'adulte sexué». Les nouvelles tendances s'orientent désormais vers une vie sexuelle épanouissante plutôt que vers les rigueurs ascétiques ${ }^{58}$.

\section{C - La famille, le social et le sexe}

Cité de Dieu, cité libérale, corporatisme, humanisme chrétien, anticléricalisme, etc., les tendances s'affrontent dans le catholicisme québécois des années 1930 à $1960^{59}$. Le discours sur l'éducation sexuelle ne peut qu'être étroitement lié aux diverses transformations sociales et institutionnelles de cette période. Tout semble se jouer autour de la famille: son intégration aux nouvelles réalités socio-économiques des années de guerre et d'après-guerre, son environnement culturel, son encadrement par l'État, ses implications dans le renouvellement du système scolaire, l'aménagement de son espace relationnel et son ajustement aux normes éducatives.

\section{1 - Le milieu culturel}

La volonté de situer de façon impérieuse l'éducation de la sexualité dans l'intimité des rapports familiaux peut se comprendre, parmi bien d'autres facettes, en regard d'un contexte de transformation culturelle bien spécifique. De plus en plus, en effet, le consensus s'établit autour du problème suivant: l'enfant, indépendamment de son milieu familial et scolaire, reçoit quantité d'informations et se forme une conception de la sexualité par un contact direct et quotidien avec le milieu ambiant. Or, ne risque-t-il pas d'échapper ainsi aux instances éducatives «légitimes»? Ä partir des différentes perceptions de la réalité moderne, se dessinent alors des figures différentes du rôle de la famille. Certains sont inquiets:

[...] c'est en dehors, et pour ne pas dire à l'encontre de leur foyer que, très souvent, ces jeunes garçons et ces jeunes filles se sont forgé des convictions morales [...]. Le mal, c'est que des parents - le petit nombre heureusement - abordent, même devant leurs enfants, une hostilité farouche envers toute discipline relative à la chasteté. D'autres, et c'est là le plus grand nombre, tout en professant, à l'occasion, de tenir aux commandements de Dieu et à l'enseignement de l'Église, affectent de ne voir de mal nulle part [...] ils tolèrent chez eux des conversations épicées, des chansons salaces, achètent, lisent et parfois laissent traîner dans la maison

58 Voir l'historique de Rita Breault, dans Les idées nouvelles viennent de la base ... (Ottawa, Serena, 1975).

Voir Jean Hamelin et Nicole Gagnon, op. cit. 
journaux, revues et magazines, non seulement étrangers à tout souci de moralité, mais même ouvertement et habilement conçus [...] de façon à flatter la sensualité des lecteurs. ${ }^{60}$

D'autres sont plus sereins; pour eux, l'éducation familiale sert de filtre aux sollicitations sensuelles du milieu:

Au moment où s'éveille son intelligence, [l'enfant] embrasse l'univers entier dans une vision magnifique, parce que colorée par son imagination débordante. Cet univers lui est révélé par l'image, par la radio, par le film. [...] En famille il a développé sa personnalité par un effort constant, renouvelé dans une liberté nécessaire à l'éducation sur mesure [...]. ${ }^{61}$

Les lieux de socialisation de la sexualité dans le contexte culturel de l'après-guerre débordent de plus en plus les anciennes méthodes d'éducation et les vieux mécanismes de contrôle. Une nouvelle culture du corps se développe, de nouvelles perceptions de la sexualité se propagent graduellement. Des publications populaires, tels Samedi, Le Petit Journal, La Revue moderne, La Revue populaire, sans parler de la radio, du cinéma, de la télévision, ni des publications qualifiées de «pornographiques», tous ces médias témoignent par leur contenu de la place grandissante de l'érotisme dans le marché de la culture de masse ${ }^{62}$.

\section{2 - La famille nouvelle}

Dans l'après-guerre, les constats de crise de la famille ou de dislocation de la société québécoise sont nombreux. On s'interroge sur la capacité de résistance de l'institution familiale ${ }^{63}$. De plus en plus, l'idéalisation et la promotion d'un modèle familial passéiste apparaissent détachées de la réalité urbaine ${ }^{64}$. Car l'immédiat après-guerre propage un nouveau style de vie domestique, axé plus intensément sur la consommation, et accentue les tendances à la recherche «privée» d'une qualité de vie. Une cellule autonome dynamique, sans cesse à l'affût de promotion et d'affirmation sociales, un foyer d'harmonie et de concorde, voilà la famille moderne, voilà l'image d'Épinal de la famille heureuse véhiculée de plus en plus par les canaux de communication modernes, par la presse à grand tirage. Tout un champ de production culturelle est accolé à ce modèle. Toute une approche de séduction convie les familles populaires à désirer les nouveaux standards, à afficher les expressions symboliques de la réussite ou à chercher les moyens d'y accéder. Ainsi, les interventions soutenant le renouveau pédago-

60 Éditorial, «Appel au sens moral chrétien», Collège et famille (avril 1952): 41-42.

61 Claudine S. Vallerand, loc. cit., 81-83.

62 Des enquêtes de l'Action catholique à la fin des années cinquante arrivent à ce constat. Voir Réal Charbonneau et Yves Ryan, Le corps humain dans la vie chrétienne (Montréal, ACC, 1958).

63 P. Lesparie, «La famille d'aujourd'hui», Relations (octobre 1949): 268-270; Fernand Dumont, «Situation de la famille canadienne-française», La Famille (mars 1952): 9-13.

64 Guy Rocher, «La famille dans la cité moderne», Service social (été 1954): 145-149. 
gique doivent-elles se comprendre dans le contexte plus global de cette «modernisation» de la famille. Une conception pédagogique qui mise sur la richesse des pulsions de l'enfant ne présuppose-t-elle pas en effet un milieu familial capable de soutenir son application? La lecture d'auteurs comme Meloche, Mercier, Vincent-Fumet, etc. nous montre bien que toute l'organisation familiale est directement en cause, tant en regard de son intégration économique et sociale que dans l'équilibre harmonieux de ses relations interpersonnelles.

\section{3 - L'État et les nouveaux intervenants}

Dans les années 1940 et 1950 , l'Église et les organisations traditionnelles en matière d'assistance publique sont de plus en plus débordées ${ }^{65}$. Malgré certaines interventions pour encadrer des besoins manifestes - oeuvres des loisirs, préparation au mariage, etc. - , l'Église s'avère inapte à régler efficacement les besoins sociaux et le potentiel humain dans son ensemble ${ }^{66}$. La modernisation de l'État, nécessaire à une bonne «gestion» des corps et de la sexualité, s'accommode souvent mal de l'arbitraire des vieux impératifs moraux. C'est pourquoi, malgré la présence persistante de structures caduques, les intervenants nouveaux dans le domaine de la vie sexuelle et familiale se développent peu à peu en nombre et en variété. L'État se voit de plus en plus pressé d'intervenir dans les domaines de protection et d'encadrement de l'enfance ${ }^{67}$ : par exemple pour les seules années 1944-45, loi de protection de la jeunesse, création du département du Bien-être social, clinique d'aide à l'enfance, allocations familiales. On souhaite le concours des «techniciens sociaux» et une organisation des services d'assistance modernisée. Médecins, psychologues, psychanalystes, criminologues, etc., s'animent d'un enthousiasme nouveau pour freiner les forces de «désintégration» de la famille et pour gérer «l'avenir de nos enfants ${ }^{68}$. Ainsi, on perçoit déjà, surtout à travers le discours des services sociaux modernes, la revue Service social plus particulièrement, une tendance à penser la famille non plus simplement comme un milieu d'éducation, mais à prendre en compte la vie familiale globale comme favorable ou non à l'épanouissement de l'enfant.

[...] les forces vives de la famille, [le service social] les voit dans la qualité morale et sociale des personnes qui la composent père, mère, enfants, chacun des enfants -; il les voit aussi, ces

\footnotetext{
1978).

Gilbert Renaud, L'éclatement de la profession en service social (Montréal, Saint-Martin,

66 Jean Hamelin et Nicole Gagnon, op. cit. 1950.

67 Voir les nombreux articles sur le sujet dans la revue Service social durant les années

68 Gonzalve Poulin, «L'avenir de nos enfants», Service social (été 1953): 54. Voir aussi L'Union médicale du Canada, Société d'hygiène et de médecine préventive de la province de Québec, La Famille et les articles de Thérèse Morisset, de Jean-Marie Martin et de Hayda Denault dans Service social.
} 
forces vives de la famille, dans le jeu harmonieux des relations familiales. Pour conserver ou restaurer ces bonnes relations indispensables, le service familial offre une variété de services concrets et surtout les services professionnels de travailleurs sociaux. ${ }^{69}$

Bien sûr, en principe, l'éducation des enfants reste encore et toujours la prérogative du milieu familial, mais de plus en plus celui-ci est enchassé dans un ensemble d'institutions et d' «intervenants sociaux» ${ }^{70}$ dont la tâche est d'évaluer et de surveiller ce potentiel en devenir. Plus que l'expression d'une stratégie bien rodée, le discours des services sociaux «modernes» nous donne, au cours des années 1950, une lecture renouvelée de la réalité sociale et familiale. Il annonce la floraison de spécialistes et de groupes d'intervention qui viendront dans les décennies suivantes fixer et diffuser les nouvelles références normatives en matière de relations familiales et de pédagogie sexuelle.

\section{4 - L'école}

Pour développer et promouvoir les idées pédagogiques modernisantes évoquées plus haut, il fallait établir une certaine harmonie entre les milieux scolaire et familial, d'où les appels répétés, dans diverses revues d'éducation, à une collaboration ${ }^{71}$. Cette préoccupation ne manque pas d'alimenter des débats, d'aviver des oppositions. Il est question d'harmoniser les méthodes et les objectifs pédagogiques mutuels en essayant d'éviter les empiétements ou les conflits de pouvoir.

C'est autour de ce bouillonnement d'idées que se manifestent, de façon très circonspecte d'abord, les premières considérations sur le rôle complémentaire de l'école et des parents dans l'éducation sexuelle des enfants. La réalité du système d'éducation québécois était, bien sûr, assez loin du débat explicite sur la pédagogie sexuelle en milieu scolaire tel qu'il apparaîtra dans les années 1960 et 1970 . Mais il ne faut pas oublier que la pédagogie catéchistique et la pastorale catholique dans son ensemble, en s'acharnant sur les questions de chasteté et en entretenant autour du sexe une quasi-obsession, faisaient du milieu scolaire un support important du dispositif entourant la sexualité. Le système d'encadrement de l'Église s'appuyait aussi bien sur l'école que sur la famille pour ramener sous son autorité morale la pédagogie de la sexualité.

Lorsqu'à travers la volonté de modernisation des années 1930 et 1940 se dessine discrètement puis s'impose graduellement une approche

69 Hayda Denault, «Service social familial et institutions», Service social, 2,4 (1952): 162.

70 Les diverses techniques d'encadrement, de même que la problématique générale adoptée par Service social nous rapprochent sensiblement, bien qu'avec certaines distorsions dues à la spécificité de l'histoire du Québec, des études de Jacques Donzelot sur l'intégration des familles aux structures économiques modernes. Voir Jacques Donzelot, La police des familles (Paris, Éditions de Minuit, 1977), 219 p.

${ }_{71}$ Un souhait souvent formulé dans les revues Collège et famille et L'École des parents. 
de la sexualité centrée plus intensivement sur le noyau familial, le système scolaire doit s'ajuster au processus. Le cheminement paraît s'opérer de façon à ce que l'école ne soit pas appelée à prendre des initiatives mais plutôt à se confiner dans un rôle complémentaire à celui des parents. Pour les intervenants catholiques ${ }^{72}$ qui abordent explicitement les questions de pédagogie sexuelle, l'idée d'une initiation «collective» est rejetée d'emblée. En raison des différences d'âge, de tempérament, de milieu familial et social, une telle approche risque, selon eux, de devenir traumatisante ou d'éveiller des pulsions subversives chez l'enfant ${ }^{73}$. On craint aussi que les enfants «bien éduqués» de l'élite ne soient soumis à une promiscuité dépréciante ${ }^{74}$. Cependant, ce qui importe davantage est de préserver l'éducation sexuelle comme une affaire d'intimité et de confidence. Dans la formation morale de l'enfant, les attitudes à tenir par rapport à ses pulsions sexuelles doivent être assimilées sous la supervision du guide parental. De façon générale, l'instituteur et l'institutrice ne sont autorisés à intervenir qu'avec la permission des parents.

Mais la modification du système d'éducation, la promotion d'une pédagogie individualisée, le souci d'harmoniser les approches éducatives des milieux familial et scolaire, toutes ces tendances viennentelles donner un rôle plus important à l'école dans l'éducation sexuelle? Les changements se font plutôt timidement. Pendant longtemps encore, les idées nouvelles et les volontés de réforme pédagogique chevauchent des pratiques et des méthodes anciennes. La famille est une institution fondamentale du catholicisme et on cherche à la défendre contre tout ce qui risquerait de l'affaiblir. Et l'éducation sexuelle, aspect important du pouvoir interne de la famille, constitue un domaine privilégié dans son renforcement.

Cependant, plus se développe le besoin d'une approche de complémentarité entre l'école et la famille, plus le milieu scolaire apparaît pour les familles populaires comme un lieu de référence et d'exigence normatives pour la réussite de l'enfant. De la même manière, l'école devient un lieu d'évaluation et de surveillance du milieu éducatif familial. À partir de là, c'est en évaluant les conséquences potentielles sur le devenir des enfants, en mesurant les degrés d'inaptitude ou d'incompétence de nombre de parents, que l'on considère le besoin possible d'une intervention de l'école en matière d'éducation sexuelle. Un autre débat s'anime à partir des années 1960: comment et en fonction de quels critères tracera-t-on une nouvelle délimitation des responsabilités et des compétences? Est-il possible de trouver un mode plus adéquat de complémentarité? Toutefois, les défenseurs de la famille entendent bien conserver leurs prérogatives.

\footnotetext{
72 Je parle surtout de ceux évoqués dans la section 2 de cet article. Entre autres Roméo Meloche, La vie ... quelle merveille!, 69. Par exemple, L.-P. Mercier, op. cit., 21 .
} 


\section{CONCLUSION}

En somme, le débat entourant la pédagogie de la sexualité ne peut s'illustrer simplement dans les termes d'une opposition tranchée entre «traditionnalistes» et «modernistes». Le processus de transformation tient plutôt, pour l'essentiel, d'une sorte d'osmose où l'on voit s'amalgamer des considérations diverses. L'ancien mode de régulation catholique s'appliquait à nommer la sexualité, à lui donner sens et réalité à travers ses prescriptions morales et à l'inscrire dans le social par des rites et des représentations spécifiques. L'enfant devait apprendre à exercer une vigilance sur lui-même, à s'avouer en offense à lui-même et au monde, mais dans un contexte précis: devant l'autorité morale «qualifiée», le prêtre, et dans le lieu secret du pardon, le confessionnal. Un rituel d'assujettissement. Mais graduellement, de façon plus ou moins sourde, les transformations et les pratiques sociales nouvelles viennent éroder les fondements de ce mode de régulation. Des tendances novatrices relancent le discours sur la sexualité et appellent inéluctablement un renouvellement des codes de référence aux valeurs acceptables. Ainsi, sous des figures diverses, dans des amalgames souvent bigarrés, ces nouvelles tendances s'insinuent en bordure du noyau dogmatique. L'éducation sexuelle apparaît de plus en plus comme une nécessité sociale et familiale des temps nouveaux. Désormais, il faut éviter d'imposer une autorité de l'intérieur mais plutôt chercher à agir du dedans, à s'immiscer jusqu'aux sources du désir et de la volonté. On doit suivre l'enfant dans la découverte de lui-même, l'amener à se délivrer de ses angoisses, à se confier. Sa formation morale doit être dirigée vers l'ennoblissement, la beauté et le respect de l'amour conjugal, vers l'affirmation de soi en accord avec la raison sociale impliquant le jeu des différenciations sexuelles. Et le tout doit dorénavant s'élaborer, en priorité, dans le lieu consacré de l'intimité, de la confiance et de la confidence: le noyau familial.

En marge de ce qui peut être perçu comme un déplacement dans la «direction» de la sexualité, se profile une tendance voulant pousser plus en profondeur les considérations sur l'enfance. Les techniques et les savoirs nouveaux doivent davantage jouer dans la découverte de soi et du monde. Le processus de formation et de réalisation de son individualité commande de miser sur l'autonomie, l'initiative et le goût de la conquête. D'où l'importance, pour la famille qui aspire à la promotion sociale, de se constituer comme un milieu éducatif épanouissant et, même, l'obligation d'acquérir les compétences nécessaires au «métier» de parent. Ce qui demande une appréciation juste de la sexualité des enfants et donc un ressourcement continuel aux lieux de production du savoir. Car, bientôt, la question n'est plus tant de dispenser un savoir «vrai» dans un langage approprié; la nouvelle normalité éducative implique une perception de la sexualité de l'enfant comme une «clef de voûte» de la personnalité. L'éducateur suscite la prise de cons- 
cience d'un secret déjà enfoui dans la personnalité de l'enfant; un travail de l'intérieur où la sexualité sert de support à l'investigation du sujet. $\mathrm{Si}$ on se réfère à l'ancienne "pédagogie de la chasteté», on se rend compte que ce n'est pas tout le support qui change mais l'ensemble des techniques reliées au savoir, à des enjeux de pouvoir et à divers types d'impératifs sociaux. Il s'agit d'un dispositif remanié, à fondement psychologique, psychanalytique et bientôt sexologique, pour que chacun puisse trouver dans les traits énigmatiques de sa personnalité, à la fois l'impression d'une singularité et l'assurance d'une appartenance collective.

Bien sûr, il ne s'agit pas de voir ces caractéristiques générales du discours comme devant engendrer aussitôt des comportements inédits ou une mise en pratique généralisée des recommandations «modernisantes». Les vieilles structures et les vieilles références religieuses, sociales et culturelles, dans le domaine de la sexualité, n'ont pas été balayées d'un coup. Dans les années 1950, le système prescriptif ancien pèse encore bien lourd. Je me suis limité, pour le moment, à caractériser les transformations et les tendances nouvelles qui se profilent dans le discours normatif sur la sexualité. J'ai évoqué un cadre normatif en éclatement dans un domaine de connaissance, de référence et de représentation aux allures éclatées. 